\title{
Spatial Correlation of Radio Waves for Multi-Antenna Applications in Indoor Multipath Environments
}

\author{
Gamantyo Hendrantoro, Puji Handayani, Achmad Mauludiyanto \\ Jurusan Teknik Elektro, Institut Teknologi Sepuluh Nopember, Kampus ITS Sukolilo, \\ Surabaya 60111, Indonesia. \\ Email: \{gamantyo, puji, maulud\}@ee.its.ac.id.
}

\begin{abstract}
This paper presents the results of a concerted effort in characterizing the spatial correlation of radio waves detected by a multi-element antenna system in indoor environments. The number of arriving paths and theirdirections are fist studied through a series of measurements. The results are then used in a simulation to obtain spatial conelation indoors. It is fund that generally an antenna array positioned near the center of the room will experience more rapidly decreasing conelation with spacing, which is a desired trait. In addition, a linear array oriented perpendicular to the length oftheroom in general shows better characteristics in terms of faster slope of correlation compared to the onein parallel with thelength oftheroom. The average correlation indoors is also found to be similar to the correlation function arising from thetwo-dimensional circularly distributed random scatterers proposed by Clarke. For practical implementation of antenna arrays in small terminals for indoor wireless applications, it is suggested that the inter-element spacing be made $0.3 \lambda$ in the least.
\end{abstract}

Keywords: antenna array, indoor radio channel

\section{INTRO DUCTION}

Signal quality and channel capacity are amongthe most essential indicators of performance of wireless communication systems. Various attemptshave been made in finding methods to maximize them simultaneously. Implementation of multi-antenna systems, either at the receiver or at the transmitter or both, is one of such efforts that have so far exhibitedgreat improvements on the two indicators [1][2]. While providing space diversity leads to improvement of error rate performance in fading channels, multiantenna systems can also render multiple channels with some degree of mutual orthogonality, which when used with spatial multiplexing scheme will considerably increase the spectral efficiency of radio channels [3][4]. Also, adaptive array antennas under the name of smart antennas have longbeen used in co-channel interference cancellation in multi-user wireless systems [5]. Achievement of the above strictly requires fulfillment of some constraints for the radio propagation channel. In particular, space diversity, spatial multiplexing, and smart antenna systems will work only when the signals on different antenna elements are uncorrelated [1][3]. Determination of spatial correlation of radio waveshas accordingly played an important role in assessment of the effectiveness of multi-antenna systems. This is true in various types of propagation environments, including both outdoor and indoor contexts.

Note: Discussion is expected before June, $1^{\text {st }} 2007$. The proper discussion will be published in Electrical Engineering Journal volume 7, number 2, September 2007.
For out door cellular systems, Clarke [6] has proposed a very well-established physical model of scatterers in which they are lined up randomly along a circumference of a circle centered at the subscriber terminal. With this model in mind, spatial autocorrelation (or temporal if mobile users are considered) of radio waves can be derived in the form of Bessel function of the first kind of zeroth order, with a ratio of distance and wavelength being the argument. A circularly located scatterers model for outdoor environments might be quite reasonable, but it does not necessarily apply indoors. Inside a room, apart from the existence of surrounding walls, floor, and ceiling, there are also randomly scatteredobjects that can act as reflectors and scatterers. This is especially true in such rooms as offices, meetingrooms, labs, classrooms, libraries, and factories [5][7].

Many papers have simply statedthat the abundance of scatterers in a room guarantees the lowcorrelation of signals on antenna elements, but they havenever gone into further detail on the form of the autocorrelation as a function of antenna separation. Thispaper reports an effort in characterizing spatial correlation of radio waves indoors in more details, with the final aim of determining a strategy to optimize receive antenna array orientation and location so as to obtain adequately uncorrelated signals on the elements. Rooms under study are assumed to be those with rectangularly-shaped horizontal layout. Thus, each of them is surrounded by four perpendicular walls of various dimensions. Both transmit and receive antennas are indoors, but they can be in separate rooms. Results are given in the form of spatial autocorrelation function of signal basebandenve- 
lopes, i.e. autocorrelation with respect to horizontal separation distance in space between two points. Aside from that, our contribution also includes recommendations on spatial orientation of the array elements with respect to the orientation of the room. The reported study is organized as follows. Firstly, a measurement campaign is conducted to obtain empirical statistics of direction of arrival (DoA) of radio waves indoors, which include statistics of number of arriving rays and angular spread. The results are subsequently used in determiningspatial aut ocorrelation of radio waves through a series of simulations using a simple 2-D ray-tracingmethod. The correlation function obtainedfrom simulation is then verified against an average correlation function obtained from direct measurements with multiple antennas indoors. In general, the study has found that the spatial aut ocorrelation of radio wavesindoors is similar to that for out door environments suggested by Clarke. More specifically, the autocorrelation is presented as a function of separation distance and is used in assessment of multi-antenna system design for indoor applications.

Following this introductory section, a physical model of the channel is described. In Section III, an experimental invest igation and a statistical analysis on the number of distinct multiple paths in an indoor environment with their respective directions of arrival are presented. The statistical results are subsequently used as the basis of a simulation study on the correlation of radio waves detectedon a number of antennas, which is verified by measurement. Finally, conclusions are drawn on the indoor spatial correlation properties of radio waves.

\section{MO DEL O F INDO O R MULTIPATH CHANNELS}

Consider a transmit-receive system operatedindoors, with a single antenna employed at the transmitter and a planar $M$-element antenna system at the receiver. Suppose the $M$-element antenna is positionedon a horizontal plane, with each element being omnidirectional. In additional, let us assume that the dimension of the multi-element antenna is sufficiently small and the location is sufficiently distant from both the transmit antenna and the surrounding wall such that the radio waves can be regardedas planar rather than spherical, even for those waves reflectingoff the walls or other objects toward the receive antenna. Considering only wave trajectories on the horizontal plane and simply assuming that the channel coherence bandwidth is greater than the transmission bandwidth, the space-time channel response can be expressed in vector form to incorporate the SIMO (single-input multi-output) nature of the channel: $\mathbf{h}(t)=\left[\begin{array}{c}h_{1}(t) \\ \mathrm{M} \\ h_{M}(t)\end{array}\right]=\sum_{n=0}^{N(t)-1} r_{n}(t) e^{j \theta_{n}(t)} \mathbf{s}\left(\psi_{n}(t)\right)$

where $t$ represents observation time, $h_{n}(t)$ represents the scalar channel response associated with the $m$-th element at time $t, r_{n}(t)$ and $\theta_{n}(t), \psi_{n}(t)$ denote the timevarying magnitude, phase and direction of arrival (DoA) of the $n$-th multipath, respectively, and $N(t)$ denotes the time-varying number of multipaths. The DoA-dependent vector $\mathbf{s}(\cdot)=\left[s_{1}(\cdot), \ldots, s_{M}(\cdot)\right]^{\mathrm{T}}$ is the so-called spatial signature of the multi-element antenna. The time variable $t$ can be easily translated into moving distance $d$ by assuming a velocity of the movement so that by assuming static conditions the channel response can also be expressedas a function of horizontal coordinate $(x, y)$ within the room:

$$
\mathbf{h}(x, y)=\sum_{n=0}^{N(x, y)-1} r_{n}(x, y) e^{j \theta_{n}(x, y)} \mathbf{s}\left(\psi_{n}(x, y)\right)
$$

with all of the other variables being as previously defined.

The number of paths representsthe number of wave trajectories with significant energy that arrive at the antenna. The magnitude and phase of a multipath altogether depend on the complex-valuedpath gain, which incorporates the effects of wave propagation through the medium as well asreflection by the walls and other objects taken into account through complex reflection coefficients. The DoA, measured in azimuth with respect to a reference zero direction, depends on the positions of the transmit andreceive antennas relative to the walls and the objects scattered around in the room. It is commonly agreedupon in the literature on multi-element antennas that thelarger the angular spread of the wave arrival, the lower the correlation of received signals among different antenna elements. The achievement of thiscondition is important in assuring optimum gain in a space diversity system or maximum accuracy in beamforming using adaptive array antenna system [5][6].

The spatial correlation of signal levels at points separated in space by a distance $\Delta$ at a position $(x, y)$ in a room can be measured and evaluated usinga linear antenna array with uniform spacing $d$, where $\Delta$ is an integer multiple of $d$. Firstly, channel response vector at that position $\mathbf{h}(x, y)$ are measuredat the output of the $M$-element antenna. The spatial correlation $\rho(\Delta, x, y)$ is subsequently computedas follows, with all of the other variables as previously defined:

$\rho(\Delta, x, y)=\sum_{m=1}^{M} \frac{1}{M-m-1} h_{m}(x, y) h_{m-\Delta / d}^{*}(x, y)$ 
The coefficient $1 /(M-m-1)$ servesto realize triangular windowing and compensates for the limitation in the number of elements. Symbol * denotes complex conjugation.

\section{NUMBER OF PATHS AND DIRECTION OF ARRIVAL}

\section{Measurement}

Characterization of number of pathsanddirection of arrival is carried out through a measurement campaign in a $3.74 \mathrm{~m} \times 9.27 \mathrm{~m}$ room with wooden benches and metal shelves [8]. The measurement system, consisting of a pair of discone antennas anda network analyzer, is shown in Figure 1. The experimental study is performed at $1.7 \mathrm{GHz}$ For detection of different multipath components arrivingfrom various azimuthal directions, the receiver employsthe synthetic array approach combined with the wellknown MUSIC (multiple signals classification) algorithm first suggested by Schmidt [9]. The synthetic planar array structure consists of a rectangularly arranged $5 \times 5$ points from which eight forward/backward $4 \times 4$ subarrays can be formedas shown in fFigure 22 , thereby allowingdetection of a maximum of eight resolvable paths [10]. The number of resolved paths itself is determinedby applyingthe MDL (minimum description length) criterion [11]. The array structure and dimension are so chosen only to fit the measurement area into theavailable spaces in the room. The floor plan of the measurement site is shown in Figure 3. All of the 28 measurement links are line-of-sight, which might imply that the detected paths will consist of one dominant direct path anda number of weaker paths, and that this condition will incur high spatial correlation of radio waves. However, the measurement results reported later show that this is not necessarily the case.

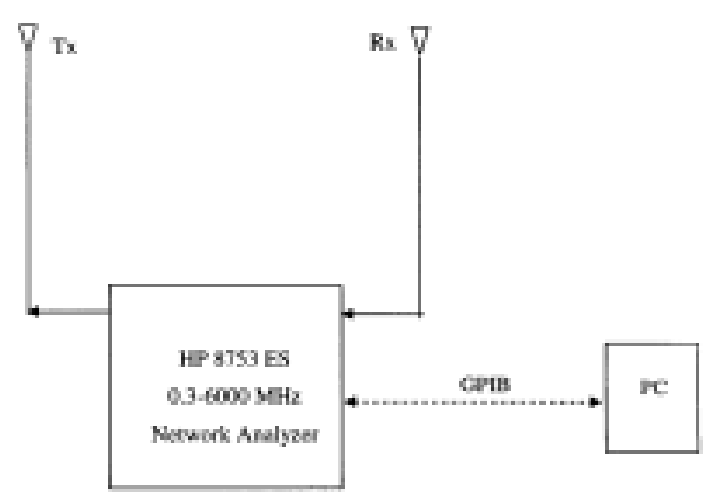

Figure 1. The measurement system

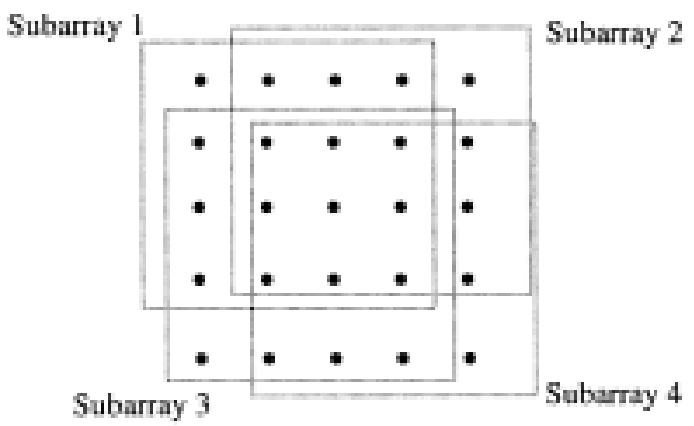

Figure 2. Structure of the synthetic planar array with its subarrays

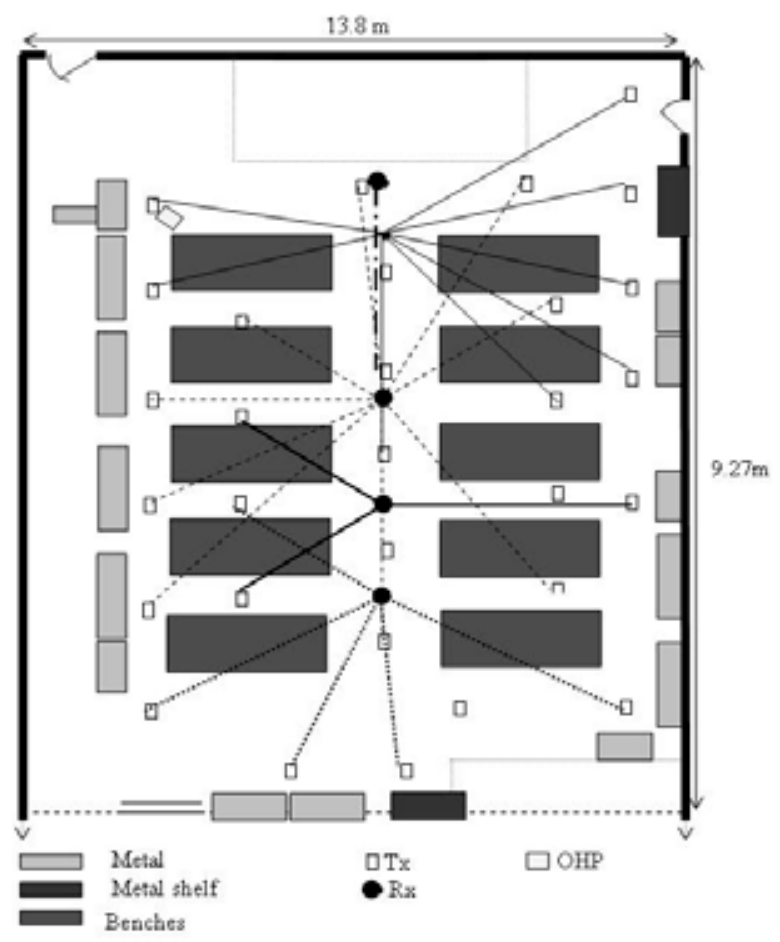

Figure 3. Floor plan and links configuration of the DoA measurement

\section{Results}

Only in one out of the 28 linksthe MUSCalgorithm fails to detect exactly eight paths, which is the maximum detectable number of pathsby the array structure employed. This leads to reasoningthat a minimum of eight radio paths occur in indoor environments. By setting the direct path to $0^{\circ}$ azimuth, histogram of the azimuthal direction of arrival of radio waves is given in Figure4. It is clear that due to line-of-sight conditions, the direct path is always in existence. The other paths, however, appear from the histogram to take random directions, with uniform distribution within therange of $-180^{\circ}$ to $180^{\circ}$ being a strong possibility. However, the existence of the direct path does not necessarily mean the received power is mostly delivered through it. Thisphenolmenon is shown by the probability density of RMS 
(root mean squared) values of the angular spread presented in Figure 5. The RMS angular spreadcan be as high as $100^{\circ}$, whereas $14 \%$ of the measured links show RMS spread of $80^{\circ}$. This is indicative of the near-uniformity of power deliverance over the whole azimuth range of radio wave arrival.

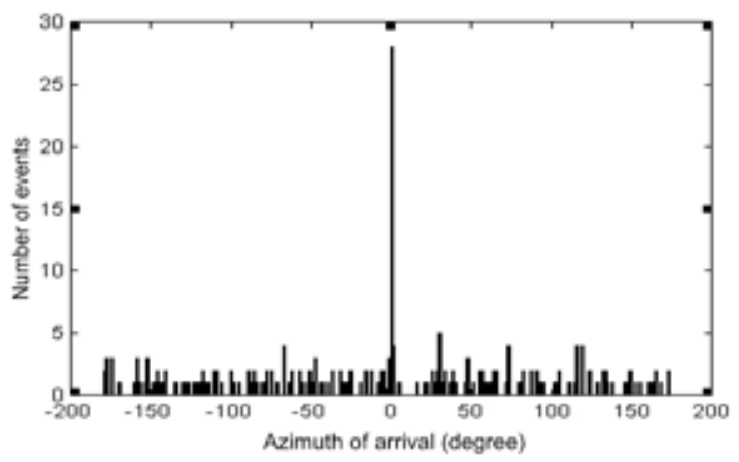

Figure 4. Histogram of azimuths of arrival

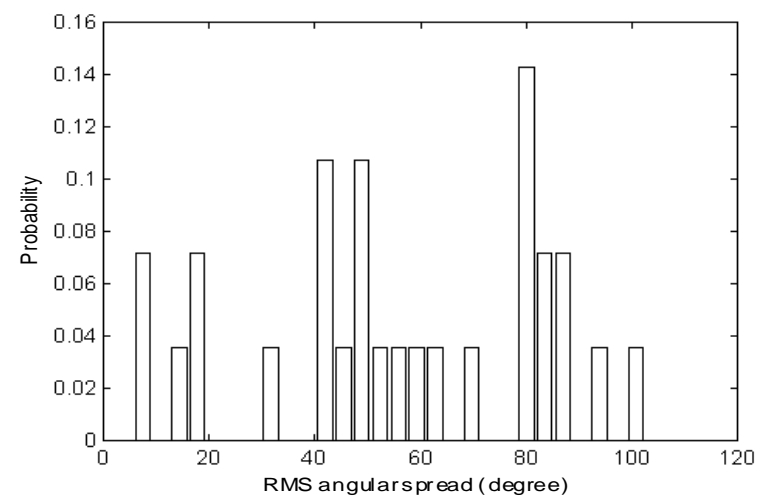

Figure 5. Probability density of RMSangular spread

\section{SPATIAL CORRELATION}

\section{DoA Statistics-BasedSimulation andMeasure- ment}

From the previous section it can besummarizedthat in indoor LOS (line-of-sight) environments astypified by the selected measurement locationstheremust be at least eight radio paths includinga direct path andat least seven other paths with uniformly distributed directions of arrival, through all of which the received power is delivered uniformly. This findingis adopted in determining the paths of radio wavesarriving from a transmitter to a group of antennas linearly arranged with half wavelength spacing [12]. A direct path is generated at all times, whereas seven others are reflected or scattered by objects surrounding the receive antennas from uniformly-distributedrandom directions. In addition, the coefficients of reflection are made random with magnitude beinguniformlydistributed in $(0,1]$ and phase beingsimilarly so in ($180^{\circ}, 180^{\circ}$. The floor plan andscenarios of the radio links are presented in Figure 6. The layout of the hypothetical room resembles arectangle of $5 \mathrm{~m} \times 10$ $\mathrm{m}$ dimension. It is considered interestingto learn the effect of both the orientation of the linear array and that of the transmit-receive direct path relative the length (the longer dimension) of the room. It is therefore decided that the simulation must look into this issue.

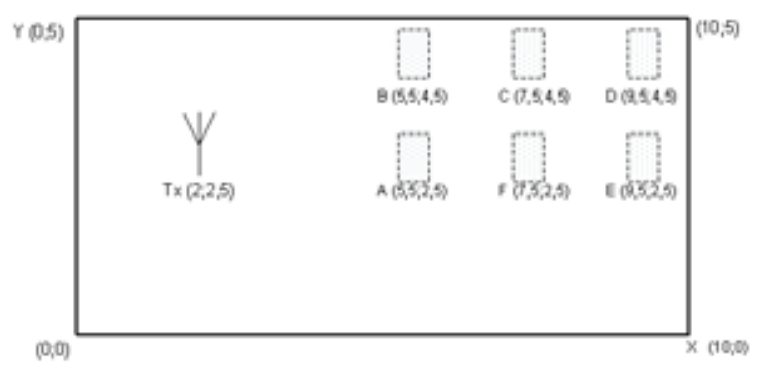

Figure 6. Scenarios of simulated radio links

Firstly, a scenario in which the transmit antennais fixed at coordinate $(2,2.5)$ is examined(see Figure 6). Four positions of the receive array are considered, namely A, B, D and E. Also, the linear array is oriented either north-south (N-S) or east-nest (E-W) at each position considered. Correlation coefficients of signals received by separate antennasare depicted in Figure 7 for the two array orientations. It can be observed that:

a. In general, when the array isorientedNorth-South the correlation coefficient decreasesrapidly with antenna separation distance. When thereceive array is at position A, the correlation isless than 0.5 for inter-element spacing greater than half a wavelength. However, for East-West orientation of the receive array, the decrease of correlation with separation distance is more gradual, for position A reaching as low as merely 0.5 at a distance of $0.4 \lambda$, rising up to 0.65 at about $0.6 \lambda$, before diving back below 0.5 at $0.7 \lambda$. For the conditions in which the receive array is located close to the wall, i.e. positions DandE, the slope is even slower, reaching about 0.75 after a wavelength separation distance.

b. When the receive array is at position A, which is approximately in the center of the room, the correlation is the lowest among those at various separation distances.

Measurements are made of signal magnitudes received by linearly arranged antennas with spacing of multiples of half a wavelength by again applying the synthetic array concept andemployingthe same measurement system and locations reported in Section III. The results are used to compute the correlation coefficients of received signals on separate antennas with the two types of orientation [13]. The coefficients at separation distances of halfandone wavelength are superposed on thesimulation results 
in Figure 7. It is clear that the measuredcoefficients are close to the corresponding values from simulation, especially for the case of position A, for both types of receive array orientation.

Figure 88 depicts the correlation coefficients for the case where the transmit antenna israndomly placed anywhere in the room, thus averagingout the effect of the fixed location in the previous simulation, with the receive array being oriented either North-South or East-West. As a result, the curves of the coefficients for different positions of receive array lie closer to one another when compared to those shown in Figure 7. The effect of different orientation on the correlation is also less significant, both N-S and E-W showing oscillation with minima near $0.4 \lambda$ and $0.9 \lambda$ andthe values almost never exceeding 0.4 for separation distances beyond $0.3 \lambda$. Superimposedon the experimental correlation curves in Figure 8 is Clarke's correlation curve [6] arising from the assumption of the two-dimensional circularly distributedrandom scatterers and which can be computed from the absolute value of the Bessel function $\mid \mathrm{J}_{0}(2 \pi d / \lambda)$.

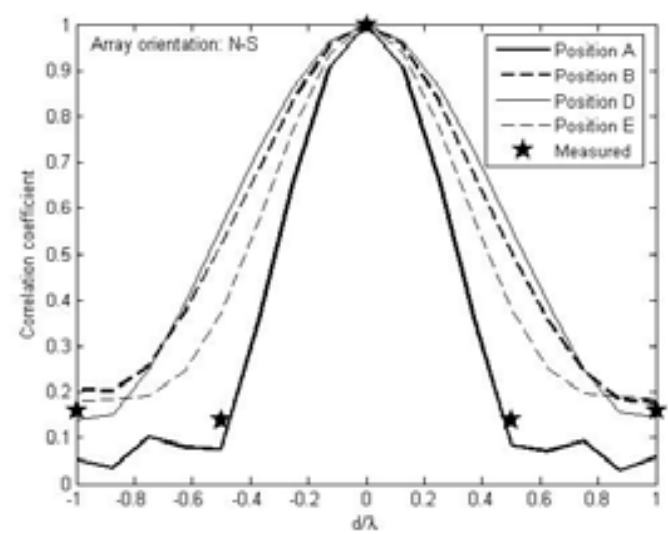

(a)

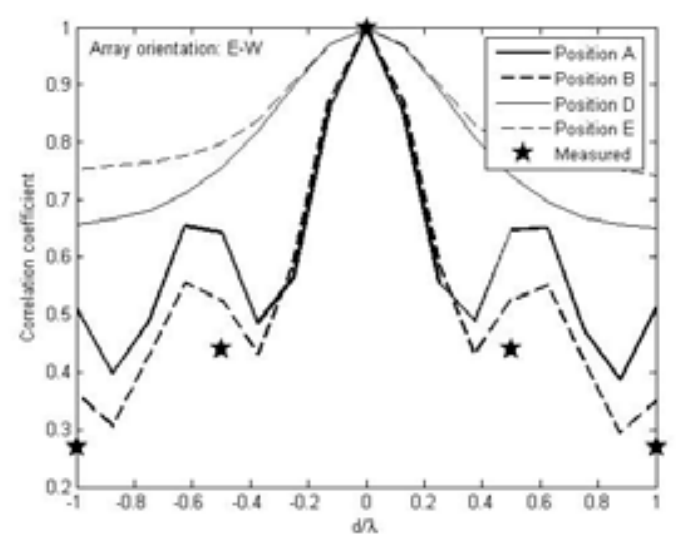

(b)

Figure 7. Correlation coefficient of receivedsignals with respect to spatial separation of antennas for a fixed transmit antennaand two orientations of receive array: (a) NorthSouth and (b) East-West

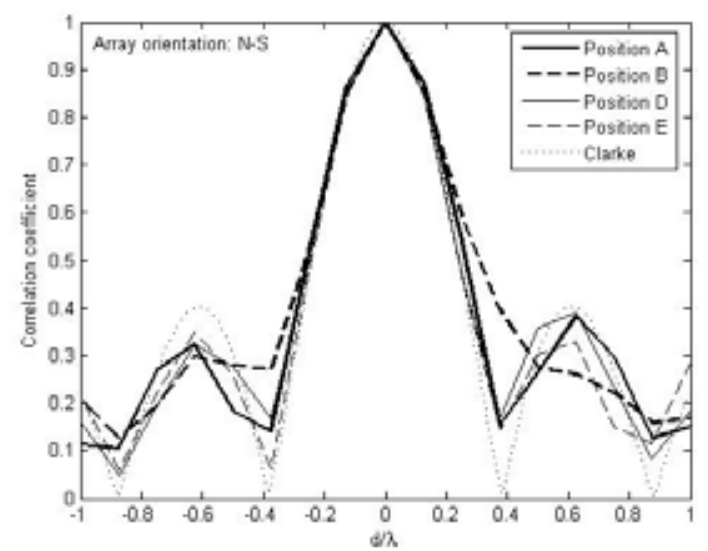

(a)

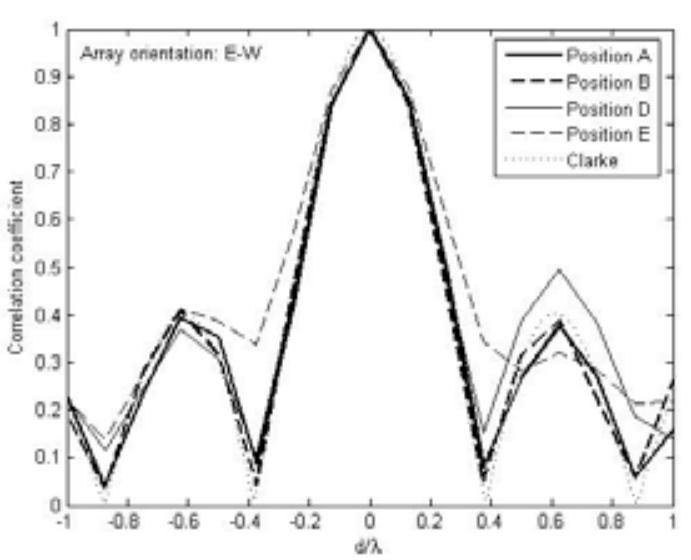

(b)

Figure 8. Correlation coefficient of receivedsignals with respect to spatial separation of antennas for various positionsof transmit antenna and two orientations of receive array: (a) North-South and (b) East-West

\section{DISCUSSION}

From the above results it can be observedthat the form of spatial correlation function depends on the position of the multi-element antenna and its orientation with respect to the length of the room and the trajectory of the LOS path. Generally the closer the array to the center of the room, the more rapidthe decline of the correlation with spacing regardess of the antenna orientation. When the antennaorientation is considered, however, the results are almost the same for all antenna positionsin the room when the linear array is orientedapproximately perpendicular to the LOS path. Whereas for antennas orientedapproximately in parallel with the LOSpath, the decreasing slope in the correlation function is slower. This observation leads to a conclusion that the best orientation of a multi-element linear array to obtain low correlation is the one perpendicular to the LOS path. 
From the evaluation of spatial comelation averaged over various positions of transmitter, it is also found that the resulting correlation function is very close to the one arising from two-dimensional circularlyspread scaterrers model proposed by Clarke. However, it can still be observed that antennas oriented perpendicular to the length of theroom results in on average more rapidly decreasing correlation with spacing compared to those orientedin parallel. It can also be concluded that the best interelement spacing for a linear array isapproximately $0.4 \lambda$, but any spacing larger than $0.3 \lambda$ generally results in correlation less than 0.4 , which is sufficiently small for all practical purposes [6]. This is very much relevant with the desireto incorporatea built-in compact array into a small terminal for indoor wireless application.

\section{CONCLUSIONS}

Spatial correlation function of radio wavesindoors has been studied through several stages. Firstly, the statistics of number of arriving waves and their directions of arrival are invest igated through a series of measurements in LOSconditions. It is found that in general at least eight paths of arrivingwaves exist at any position indoors includingthe LOSpath and that despite the LOS conditionsthe energy of the arriving waves is spread over a large angular spread, promising a low spatial correlation.

The results are then used in simulation to study the correlation function, which is then verifiedby field measurement, with the final aim of determininga strategy to optimize receive antenna array orientation and location so as to obtain adequately uncorrelated signals on the elements. The findingsinclude that in general an array positioned near the center of the room will experience more rapidly decreasing correlation with spacing, which is a desiredtrait. In addition, a linear array orientedperpendicular to the length of the room in general shows better characteristics in terms of faster slope of comelation compared to the one in parallel with the length of the room. Finally, for practical implementation of antenna arrays in small terminals for indoor wireless applications, it is suggested that the inter-element spacing be made $0.3 \lambda$ in the least.

\section{Acknowledgement}

A number of fellow faculties and students of the Department of Electrical Engineering of Institut Teknologi Sepuluh Nopember were involved in the measurement campaigns and subsequent analyses, including Dr. A. Affandi, Mr. Suwadi, Mr. Endro- yono, Ms. N. A. Siswandari, and Mr. H. Hermitch. Their contribution is gratefully acknowledged.

\section{REFERENCES}

[1] J. H. Winters, "On the Capacity of Radio Communication Systems with Diversity in a Rayleigh Fading Environment", IEEEJoumal on Selected Areas in Communications, vol. 5, no. 5, June 1987, pp. 871-878.

[2] D. Gesbert, M. Shafi, D. Shiu, P. J. Smith, A. Naguib, "From Theory to Practice: An Overview of Space-Time Coded MIMO Wireless Systems", IEEE Journal on Selected Areas in Communications, vol. 21, no. 3, April 2003, pp. 281-302.

[3] G. Foschini, "Layered Space-TimeArchitecture for Wireless Communication in a Fading Environment When Using Multi-Element Antennas", Bell Labs Technical Joumal, 1996.

[4] J. B. Andersen, "Array Gain and Capacity for Known Random Channels with Multiple Element Arrays at Both Ends', IEEE Joumalon Selected Areas in Communications, vol. 18, no, 11, November 2000, pp. 2172-2178.

[5] J. C. Liberti, T. S. Rappaport, Smant Antennasfor Wireless Communications, Prentice-Hall 1999.

[6] W. C. Jakes, Microwave Mobile Communications, IEEE Press, 1974.

[7] G. Hendrantoro, D. D. Falconer, M. S. ElTanany, "Characterization of $29.5 \mathrm{GHz}$ Broadband Indoor Radio Channels Using a Steerable Receiving Antenna", Wireless Communications Conference, Boulder Colorado, August 1997, pp. 205-209.

[8] P. Handayani, Statistical Characterization of Radiowave Angles of Arrival at $1.7 \mathrm{GHz}$ in Indoor Environment [in Indonesian], Master's Thesis, Institut Teknologi Sepuluh Nopember, Surabaya, 2003.

[9] R. O. Schmidt, "Multiple Emitter Location and Signal Parameter Estimation", IEEE Trans. on Antennas and Prop, March 1996.

[10] Y.-M. Chen, "On Spatial Smoothingfor TwoDimensional Direction of Arrival Estimation of Coherent Signals", IEEE Trans. on Signal Processing, vol. 45, No.7, July 1997.

[11] M. Wax and I. Ziskind, "Detection of The Number of Coherent Signals by The MDL Principle", IEEE Trans. on Acoustics, Speech, and Signal Processing, vol. 37, No. 8, August 1989.

[12] H. Hermitch, Spatial Correlation Coefficients of Radiowaves and Diversity Gain in Indoor Environments for Applicationsin Multi-Antenna Communication Systems [in Indonesian], Under- 
graduate Final Project, Institut Teknologi Sepur luh Nopember, Surabaya, 2003.

[13] N. A. Siswandari, Spatial Comelation Analysis of Indoor Radio Propagation Channelsat $1.7 \mathrm{GHz}$ Using a Synthetic Planar Array Antenna [in Indonesian], Master's Thesis, Institut Teknologi Sepuluh Nopember, Surabaya, 2003. 